\title{
Re: Fluorescence In Situ Hybridization Detects Increased Sperm Aneuploidy in Men with Recurrent Pregnancy Loss
}

\author{
Ranjith Ramasamy, Jason M. Scovell, Jason R. Kovac, Peter J. Cook, Dolores J. Lamb, Larry Lipshultz \\ Baylor College of Medicine, Department of Urology, Houston, Texas
}

Fertility and Sterility 2015;103:906-909.

\section{EDITORIAL COMMENT}

Male factor infertility can be overcome with the use of assisted reproductive technologies and for this purpose the mostly intracytoplasmic sperm injection (ICSI) was used. Although using sperm from men with relatively normal semen parameters with high-tech methods, many couples fail to achieve pregnancy or face recurrent pregnancy loss (RPL). In this study, the authors tried to find an answer for potential causes of RPL and in vitro fertilization (IVF) failure by using fluorescence in situ hybridization (FISH) analysis. FISH analysis was used to detect numerical abnormalities in sex chromosomes $(X, Y)$ and autosomes $(13,18,21)$ in ejaculated sperm. Significantly higher percentage of sperm aneuploidy was found in men with RPL within the sex chromosomes and chromosomes 18,13 and 21. Although men with normal sperm parameter, $40 \%$ of abnormal sperm aneuploidy was found in all the chromosomes analyzed. In addition to that, men with abnormal sperm density and motility had a higher percentage of sex chromosome aneuploidy than men with normal density and motility. In conclusion, sperm FISH analysis can be suggested in men with RPL and normal sperm density/motility to understand the reason of pregnancy failure. Also, this study showed that men with oligoasthenoteratozoospermia (OAT) might have a greater percentage of sperm aneuploidy compared to those with normal sperm parameters.

\section{Re: A Population-Based Analysis of Contemporary Rates of Reoperation for Penile Prosthesis Procedures}

\author{
S. Grewal, J. Vetter, S. B. Brandes, S. A. Strope \\ Washington University in St. Louis, Department of Surgery, Division of Urology, St. Louis, Missouri
}

Urology 2014;84:112-116.

\section{EDITORIAL COMMENT}

A database of 2.263 penile implant cases (1824 inflatable and 439 semi-rigid) was analyzed for factors associated with reoperation. Both prosthesis had a similar reoperation rate (nearly 7\%), although noninfectious reoperations were slightly more common with inflatable devices. The reoperation rate secondary to infectious complications was $4.5 \%$ for semi-rigid and $3.23 \%$ inflatable group, which was not significantly different. Inflatable prosthesis is well tolerated by penile tissue but mechanical problems were seen more likely. 